\title{
The influence of alternative data smoothing prediction techniques on the performance of a two-stage short-term urban travel time prediction
} \section{framework}

Fangce Guo, Rajesh Krishnan, and John Polak

Centre for Transport Studies, Imperial College London, London, UK

\begin{abstract}
:
This paper investigates the impact of alternative data smoothing and traffic prediction methods on the accuracy of the performance of a two-stage short-term urban travel time prediction framework. Using this framework, we test the influence of the combination of two different data smoothing and four different prediction methods using travel time data from two substantially different urban traffic environments and under both normal and abnormal conditions. This constitutes the most comprehensive empirical evaluation of the joint influence of smoothing and predictor choice to date. The results indicate that the use of data smoothing improves prediction accuracy regardless of the prediction method used and that this is true in different traffic environments and during both normal and abnormal (incident) conditions. Moreover, the use of data smoothing in general has a much greater influence on prediction performance than the choice of specific prediction method and this is independent of the specific smoothing method used. In normal traffic conditions the different prediction methods produce broadly similar results but under abnormal conditions, lazy learning methods emerge as superior.
\end{abstract}

Keywords Intelligent transportation systems (ITS); short-term traffic prediction; data smoothing; machine learning method

\section{INTRODUCTION}

The field of Intelligent Transport Systems (ITS) has developed rapidly over the last two decades, driven by the growing need for better transport network management strategies and by continuing improvements in computing power. An important class of ITS services use traffic state information such as traffic flow, speed and travel time obtained from various traffic sensors to monitor and control transport networks and also to provide information to travellers. Increasingly, these services aim to be proactive rather than reactive, and consequently require methods that are capable of providing reliable short-term predictions of key traffic state variables, such as travel time.

Short-term traffic prediction can be defined as the process of estimating the anticipated traffic conditions in the short-term future given historical and current traffic information (Vlahogianni et al., 2004). In this paper, the phrase short-term is used to refer to a time horizon of up to one hour. The existing literature shows that a wide range of methods have been used in short-term traffic prediction, including both statistical methods and machine learning methods. However, the accuracy of the traffic prediction using a given prediction model will depend not only on the prediction model itself, but also on the characteristics of the underlying data generation and measurement processes. These considerations are particularly important in urban areas, where the traffic state is subject to intermittent disruptions (e.g., accidents, breakdowns, roadworks, demonstrations and special events) that can temporarily change the underlying dynamics of the data generation process and where traffic sensor systems are often deployed in hostile conditions that result in noisy and incomplete data (Krishnan, 2008; Robinson, 2005).

The challenges of short-term prediction under conditions intermittent disruptions leading to temporarily abnormal conditions together with noisy measurement processes are of course 
not unique to transport and occur in a many other domains, for example hydrology (see Sivapragasam, Liong, \& Pasha 2001). An approach that has been demonstrated to improve prediction performance in a number of domains is to pre-process input sensor data, using data smoothing and de-nosing techniques, prior to the application of formal prediction models. Although researchers have recently begun exploring the potential benefits of data smoothing techniques in short-term traffic prediction, the field is still at an early stage of development and empirical experience is limited.

Against this background, the overall aim of this paper is to examine the impact of alternative data smoothing techniques on the accuracy of the short-term traffic prediction of link travel time, taking into consideration a number of different prediction methods and data from in a number of different traffic conditions, including both normal and abnormal (disrupted) conditions. The next section provides a brief overview of the relevant existing literature covering both prediction and data smoothing methods. The third section sets out a two-stage prediction framework, comprises a data smoothing stage followed by a prediction stage, and describes the specific techniques used in the implementation of each stage. The fourth section describes the data and the experimental design used in the empirical tests. This design comprises link travel time data from two UK locations with very different conditions; central London and the smaller freestanding town of Maidstone, in each case collected under both normal and abnormal conditions. The fifth section presents the results of the empirical comparison and the final section discusses the overall conclusions of the work.

\section{EXISTING RELATED WORK}

A wide range of traffic prediction methods are available in the literature, using a number of different statistical and machine learning approaches. Some notable early examples include the work of Ahmed and Cook (1979), who applied Auto-Regressive Integrated Moving Average (ARIMA) time series models (Box \& Jenkins, 1970) to the prediction of freeway traffic flow and the work of Okutani and Stephanedes (1984) who developed a Kalman filter based approach to prediction of traffic flow. More recently, machine learning methods, such as Neural Networks (NN) (e.g., Dougherty, 1995; Zhang \& Zhang, 2016) and the k-Nearest Neighbour (kNN) (e.g., Davis \& Nihan, 1991; Yoon \& Chang 2014) and traffic flow models, such as Cell Transmission Model (CTM) (Szeto et al., 2009) and spatio-temporal Kalman Filter (Wu et al., 2016) have been used for flow and travel time prediction. The most recent generation of machine learning tools applied to short-term traffic forecasting include Support Vector Regression (SVR) (e.g., Wu et al., 2004), Grey system Model (GM) (e.g., Guo et al, 2013) and particle filtering (e.g. Chen \& Rakha 2014).

A problem that affects all methods of short-term prediction in this field is the prevalence of noisy and incomplete data, arising from pervasive sampling and measurement errors in all commonly used sensor systems. In recent years, researchers have begun to investigate the effect of formal data smoothing techniques to reduce the effect of noisy information and increasing the traffic prediction accuracy. Xiao et al. (2003) developed a perdiction framework based on NN techniques linked to a wavelet smoother to forecaset 5-minute ahead traffic speed for a freeway link in Houston. Later, Xie et al. (2007) used wavelet deconposition analysis with the Kalman filter method to predict 5-minute future traffic volume on a highway in Seattle. Their results showed that the smoothed Kalman filter method outperform the direct Kalman filter method without smoothing stage in terms of mean absolute percentage error and root mean square error. Jin et al. (2007) used Principle Component Analysis (PCA) as a smoother with the SVR method to predict traffic flow data collected from the centre of Beijing. They compared the prediction results of proposed smoothed model with those using NN and ARIMA 
predictors. The results showed that the smoothed SVR model had the smallest errors of all the methods.

These studies have demonstrated that data smoothing/de-noising used in conjunction with specific prediction methods can improve the accuracy of short-term traffic prediction. However, some of these studies (e.g., Xiao et al., 2003; Xie et al., 2007) directly used the smoothed data after de-noising rather than original noisy data and discarded the noisy series in the process of training and testing. In real problems, traffic data are never noise free. The smoothed data describe the trend of traffic data and the noisy residuals reflect the details of traffic data series. Thus, both the smoothed trend and the noisy residuals should be considered in practice. Moreover, none of these studies have attempted to systematically evaluate the impact of data smoothing/de-noising on the accuracy of short-term traffic prediction across a number of different prediction methods.

In this paper we will systematically investigate and generalise the impact of data smoothing/de-noising techniques on traffic prediction accuracy across a number of very different machine learning methods and under both normal and abnormal traffic conditions. The proposed two-stage framework uses original noisy traffic data as inputs and takes both smoothed series and residuals into account.

\section{PREDICTION FRAMEWORK}

This paper uses the general two-stage framework for short-term traffic prediction introduced by Guo et al. (2013) in the context of traffic flow prediction. In this paper, the framework is applied to the related problem of link travel time prediction; link travel time is defined as the average travel time of all the vehicles that exit the link during a given time period. In this two-stage framework, the first stage is to smooth traffic data in order to extract the smoothed component and residuals from noisy data. The second stage involves the use of a machine learning technique that can extract the relationship between current and future smoothed traffic variables. In addition, the historic average residual value is used as the predicted residual component. The predicted values of smoothed and residual components are combined to predict the future values of noisy traffic variables. The framework thus essentially adds a data pre-processing stage to prediction models commonly found in the literature, while also making use of residual values after smoothing.

The premise behind data smoothing pre-processing step is that the subsequent machine learning models will be able to learn the patterns in data better after de-noising. A distinctive element of the Guo et al. (2013) framework which distinguishes it from most other similar approaches in the transport literature is that it uses both the smoothed series information and the residual information for the prediction of noisy data. The usefulness of residual information within such prediction frameworks has been demonstrated in other domains, for example image processing (see Brunet et al., 2009) as well as in the context of flow prediction by Guo et al. (2013), and it is surprising that the transport literature has largely overlooked this issue to date.

The traffic prediction framework is illustrated in Figure 1. In the application of traffic prediction, two types of data series inputs are assumed - historical $\left(x_{h}\right)$ and currently observed $\left(x_{c}\right)$ traffic data. Historical traffic data are used for training, and currently observed traffic data informs on the current traffic state. In the first data smoothing step, the historical traffic data $x_{h}$ is decomposed into a smoothed series $x_{h_{-} s}$ and its residual $x_{h_{-} r}$ in an offline process. At the same time, the estimated residual series $\hat{x}_{h_{-} r}$ is defined using the historical average value of the residual $x_{h_{-} r}$. In the online process, the data smoothing extracts a smoothed component $x_{c_{-} s}$ from the observed traffic data (and discards the residual). The final prediction result is the sum of the predicted smoothed $\hat{x}_{c_{-} s}$ and the estimated residual $\hat{x}_{h_{-} r}$, based on the historical data. The machine learning methods are not efficient to forecast very irregular data patters such as 
residuals. Thus, the framework only uses the machine learning methods to predict the smoothed series and the residual part is characterised by an averaged analysis.

\section{Data Smoothing}

\section{Introduction}

The proposed two-stage framework is generic and any suitable data smoothing method can be used within the framework. A large number of data smoothing methods are available in the literature. These include the wavelet transform (Xie et al., 2007), Savitzky-Golay smoothing (Barclay et al., 1997), Fourier filtering (Kosarev \& Pantos, 1983) and Singular Spectrum Analysis (SSA) (Golyandina et al., 2001). This paper uses both SSA and wavelet decomposition since they are widely used in a variety of different applications and are good representatives of the current state of the art in data smoothing (Chang \& Fan, 2008; Hassani, 2007) .

\section{SSA data smoothing}

Singular Spectrum Analysis (SSA) is a data smoothing method used in the analysis of time series. It is widely used in many fields, such as hydrology (e.g., Sivapragasam et al., 2001) and atmospheric and geophysical research (e.g., Ghil \& Vautard, 1991). SSA is a model-free, adaptive noise-reduction algorithm based on the Karhunen-Loeve transform (Sivapragasam et al., 2001). It can also be used as a data de-noising method by decomposing an original time series to a smoothed series and a (residual) noise series (Hassani, 2007). Mineva and Popivanov (1996) present a comprehensive description and discussion of the SSA method and identify a number of advantages of SSA compared to other data smoothing techniques. These advantages include the ability to characterise both trend and oscillatory components, the capability to reduce local noise, enhance pattern recognition and computational efficiently.

The basic idea of the SSA approach is to undertake a spectral analysis of the raw input data in order to separate out high frequency "noisy" components thus allowing the remaining components to be reconstructed into a smoothed version of the original series. The SSA algorithm is typically implemented in the following four steps (Golyandina et al., 2001):

- Step 1: This step is an embedding step that transfers the original one-dimensional time series $X$ to a multidimensional series $T_{X}$, which can form the trajectory matrix.

- Step 2: This step uses Singular Value Decomposition (SVD) to change the trajectory matrix formed in the Step 1 into a decomposed trajectory matrix. The trajectory matrix $T_{X}$ can be written as

$$
T_{X}=\sum_{i=1}^{r} u_{i} \sigma_{i} v_{i}^{T}
$$

where $u_{i}$ and $v_{i}$ are the left and right eigenvectors of the trajectory matrix and $r$ is the rank of $T_{X}$. The element $u_{i} \sigma_{i} v_{i}^{T}$ is called the $i^{t h}$ eigentriple of the SVD.

- Step 3: The decomposed trajectory matrix will be reconstructed in this step. This step is a grouping step and corresponds to splitting the matrices, computed at the SVD step, into several groups and summing the matrices within each group. The expansion of $T_{X}$ can be written as

$$
T_{X}=f_{S V D}\left(T_{1}, T_{2}, \ldots, T_{r}\right)
$$

- Step 4: A new time series is created by the grouped matrices in Step 3. The corresponding operation is called diagonal averaging. It is a linear operation and maps 
the trajectory matrix of the initial series into the initial series itself. In this way, a decomposition of the initial series into several additive components can be obtained.

\section{Wavelet decomposition}

Wavelet transform was proposed as an analysis method for extracting local-frequency information from a signal (Daubechies, 1990) and is widely used as a data smoothing method (Torrence \& Compo, 1998). Discrete Wavelet Transform (DWT) used in this paper can decompose the original signal into approximations and details. The original data $x(t)$ can be decomposed and reconstructed using the following equation

$$
x(t)=\sum_{m=1}^{L}\left[\sum_{k=-\infty}^{\infty} D_{m}(k) \psi_{m, k}(t)+\sum_{k=-\infty}^{\infty} A_{l}(k) \phi_{l, k}(t)\right]
$$

where $\psi_{m, k}$ is the discrete wavelet function, $\phi_{l, k}$ is discrete scaling function, $D_{m}$ is the details at scale $2^{m}$ and $A_{l}$ is the approximations at scale $2^{l} . D_{m}$ and $A_{l}$ are obtained using the scaling and wavelet filters.

In the first step of the proposed prediction framework, SSA and Wavelet transform are used in the data smoothing step. The original traffic time series is divided into two parts in this step: the smoothed series and the residuals. For example, Figure 2 illustrates the decomposition of traffic flow data into the smoothed part and its residuals using SSA.

\section{Machine learning methods used for prediction}

Four different machine learning methods are used in this paper to investigate the hypothesis that the proposed two-stage framework improves prediction accuracy. Machine learning methods can consider traffic processes as black boxes and learn the relationship between inputs in order to predict traffic variables directly. Not only are these methods less complicated and burdensome than simulated methods to implement for prediction, they may also potentially enable the prediction process to adapt more easily to normal or abnormal traffic regimes. The prediction methods used are k-Nearest Neighbour (kNN), Grey system Model (GM), Random Forests (RF) and Support Vector Regression (SVR). These are commonly used but quite different techniques for short-term traffic prediction (Vlahogianni et al., 2004). A brief overview of each of these methods is presented below.

\section{The k-Nearest Neighbour method (kNN)}

The kNN method is a lazy learning method that does not involve any explicit model construction (Hastie et al., 2001). The kNN method locates a number of observations (termed the nearest neighbours) from an historical dataset and then predicts future data based on this nearest neighbour subset. In traffic prediction applications, this nearest neighbour set comprises historical traffic data that are, in some appropriate sense, similar to the observed traffic state.

The kNN based prediction method can be decomposed into three fundamental components: a database of past observations, a neighbourhood search procedure and a prediction function. The search procedure finds the nearest neighbours, which are the historical observations that are most similar to the current conditions. The nearest neighbours then become the inputs to the forecast function so that it may generate a prediction.

\section{Grey system Model (GM)}

Grey system Model (GM) based methods (Deng, 1989) predict the future values of a time series based only on a set of the recently observed data, where the observation process depends on the window size of the predictor (Kayacan et al., 2010). It is assumed that all data values to 
be used in grey models are positive, and the sampling frequency of the time series is fixed. In their simplest form, GM can be viewed as a sophisticated type of local curve fitting technique. The GM model constructs a differential equation to describe the unknown system. The output of GM can be calculated by solving the differential equation. GM methods are widely used in the financial domain (Kayacan et al., 2010), but have only recently been used in the transport domain by Guo et al. (2013), who also provide a more detailed statement of the method.

\section{Random Forests (RF)}

The random forests based method was first introduced by Breiman (2001) as a statistical learning method for use with high-dimensional classification and regression problems, where classification is used to model categorical variables and regression is used to predict continuous variables.

RF grows an ensemble of trees using training data. Unlike standard classification trees, RF employs randomness when selecting a variable to split each tree and each node. Each node is split using the best split-point among a subset of predictors randomly chosen at that node, rather than, as would more conventionally be the case, choosing the best one among all predictors (Liaw \& Wiener, 2002). The random parameter vector is used to determine the growth of the trees and to calculate the split-points at each node. For regression, the prediction of random forests at a new point is the average of all corresponding trees. More details of RF can be found in Verikas, Gelzinis, and Bacauskiene (2011).

\section{Support Vector Regression (SVR)}

Support Vector Regression (SVR) implements the structural risk minimisation principle from computational learning theory (Vapnik, 1995). The basic idea of SVR methods is to map the input data into a high dimensional feature space and then calculate a linear regression in the high dimensional space. Therefore, a non-linear regression in a low dimensional space can be transferred to a linear regression in a high dimensional feature space. SVR methods have been successfully applied to a number of data prediction applications ranging from financial data to traffic data.

\section{EXPERIMENTAL DESIGN}

The objective of the experiment is to quantify the effects on prediction accuracy of using the proposed two-stage prediction framework, including explicit data smoothing and de-nosing. In order to achieve this objective, prediction accuracy using each of the machine learning methods is compared against the same method when applied within the proposed prediction framework. The context of this paper for short-term traffic prediction is the urban road network in the UK. Two quite different testing locations in urban areas are selected to examine the location transferability of the proposed traffic prediction framework; central London and the freestanding town Maidstone. Both these locations experience traffic congestion during both morning and evening periods but have very different network configurations, traffic control arrangements and traffic composition.

\section{Scenario 1: Prediction under normal conditions}

\section{London case}

All link travel time data from London was obtained from the London Congestion Analysis Project (LCAP) (TfL, 2010). LCAP is operated by Transport for London (TfL) to capture and store link travel time data in London based on Automatic Number Plate Recognition (ANPR) camera data. LCAP calculates the averaged link travel time data at 5-minute intervals; the travel 
time during a 5-minute period is the average travel time of all the vehicles that exit the link during that 5-minute period after the removal of outliers. The data used in this paper comes from a $5.63 \mathrm{~km}$ section (Link 1309) of the A40, which is a dual carriageway with limited access that serves as one of London's major radial corridors (see Figure 3). Link 1309 is monitored by a pair of ANPR cameras. The direction of travel is from west to east.

Travel time data for a period of three months between January and March 2011 is divided into two datasets. Training data is from $3^{\text {rd }}$ January 2011 to $17^{\text {th }}$ March 2011; while testing data is from $18^{\text {th }}$ March 2011 to $31^{\text {st }}$ March 2011. Since the focus in on weekdays, weekend data is eliminated. Both training dataset and testing dataset are concerned with normal traffic conditions.

\section{Maidstone case}

Travel time data from Maidstone was provided by Kent County Council (KCC). The link travel time data in Maidstone was monitored by ANPR. The data used in this paper comes from a $6.4 \mathrm{~km}$ section (Link 99AL0005D) of the A229, which comprises mixture of single and carriageway road with extensive turning and signal control (see Figure 4). The training data was collected from $1^{\text {st }}$ June 2011 to $24^{\text {th }}$ August 2011 . The testing data was collected on $25^{\text {th }}$ August 2011. Only weekday travel time data under normal traffic conditions was included in this dataset. Due to the accessibility of travel time data from Link 99AL0005D, traffic time data from the morning peak period (07:00-10:00) and evening peak period (16:00-19:00) was extracted in the training dataset.

\section{Scenario 2: Prediction under abnormal conditions}

Scenario 2 consists of known traffic incidents as well as normal traffic conditions in London and Maidstone.

\section{London case}

The data from London used in this scenario was from the same location as those described in Scenario 1. Link travel time data from link 1309 of the A40 road in central London was extracted in this experiment. Traffic data under normal traffic conditions is used in the training dataset while the testing dataset consists of known traffic incidents. Training data that are concerned with normal traffic conditions is from weekdays during October, November and December 2010; testing data is from $21^{\text {st }}$ December 2010. One lane was blocked on Western Avenue eastbound on the testing day because of a broken down vehicle. Information about abnormal traffic conditions was also directly provided by TfL, including event date, start time, end time, category, location and severity. Detailed information of this abnormal event is as below:

\footnotetext{
$\checkmark$ Abnormal event date: $21^{\text {st }}$ Dec 2010

$\checkmark$ Abnormal event period: About 45 minutes from 17:57 to 18:40

$\checkmark$ Abnormal event category: Broken down vehicle

$\checkmark$ Abnormal event location: Western Avenue
}

\section{Maidstone case}

The data used in the Maidstone case in this scenario was from the same location as those described in Scenario 1. An accident happened on Link 99AL0005D in the Maidstone area of Kent on $26^{\text {th }}$ August 2011, which caused the northbound A229 Royal Engineers Road to become partially blocked. This abnormal information was directly provided by $\mathrm{KCC}$, including event date, start time, end time, category, location and severity. Detailed information of this abnormal event is as below: 
$\checkmark$ Abnormal event date: $26^{\text {th }}$ Aug 2011

$\checkmark$ Abnormal event period: About 30 minutes from 16:23 to 16:51

$\checkmark$ Abnormal event category: Accident

$\checkmark$ Abnormal event location: A229 Royal Engineers Road, Maidstone area

The training data used in this scenario was collected from $1^{\text {st }}$ June 2011 to $25^{\text {th }}$ August 2011.

\section{Key parameter settings of the machine learning methods}

\section{Key parameters of $k N N$}

There are three key design parameters applying the kNN based method: distance metric, choice of $\mathrm{k}$ and prediction function. The distance metric is used to calculate the distance between two feature vectors. The distance metric may influence the results of data classification (Short \& Fukunaga, 1981). However, the distance metric in time series prediction with large training datasets is not the most significant component. Euclidean distance as one of the most common method in prediction to calculate the distance is used in this paper. Too small a value of $k$ will filter out relevant neighbours; too big a value of $k$ will introduce noise and weaken the prediction. It found that the optimal value of is data-dependent, and it usually grows with the sample size and variability in data (Stone, 1977). Traffic prediction accuracy varies according to the type of prediction function (Smith et al., 2002). The trial and error method described in Guo et al. (2012) is used for the optimisation of parameter of $\mathrm{kNN}$ in this paper. The number of neighbours $(k)$ is set to 30 and weighted by inverse of distance is selected as the prediction function.

\section{Key parameters of GM}

GM is selected in this paper because of its reduction of the dependency on method training and parameter optimisation. In grey system theory, a grey system $\operatorname{GM}(n, m)$ can dynamically update parameters based on the relationship between feature vectors. $\operatorname{In} \operatorname{GM}(n, m), n$ is the order of the differential equation and $\mathrm{m}$ is the number of variables. Various types of grey system models can be found in the literature; however, a $\operatorname{GM}(1,1)$ model is most commonly used because of its performance and computational efficiency, which are important design parameters in practice.

\section{Key parameters of $R F$}

The most important RF parameter that needs to be pre-determined in the optimisation process is the number of trees in the forest. The trial and error method is used to optimise this parameter in this paper. After optimising, the value of number of trees is set to 490 .

\section{Key parameters of SVR}

There are three key parameters in the implantation of SVR: kernel function, capacity value $C$ of SVR and the width of $\varepsilon$-intsensitivity area for fitting the training dataset. The selection of kernel function is usually based on a priori knowledge of application domains (Chapelle \& Vapnik, 1999). In this paper, we deploy one of the most widely used functions in transport engineering, a Radial Basis Function (RBF) kernel. Several methods of selection of SVR parameters is described in Cherkassky and Ma (2004). Cross-validation for parameter selection method is used in this paper to optimise SVR parameters described above. In the optimisation process, the SVR parameters are chosen as the ones that minimise the Mean Absolute Percentage Error (MAPE). The value $C$ is set to 80 and the value of $\varepsilon$ is 0.05 .

\section{Quantification of prediction accuracy}


The prediction accuracy is evaluated using three criteria, namely Mean Percentage Error (MPE), Mean Absolute Percentage Error (MAPE) and Root Mean Square Error (RMSE). MPE is the average of the percentage errors for a given dataset during a specific period and is used to calculate prediction bias. MAPE calculates the average of the absolute difference between predicted and actual values. Both positive and negative predictive errors can be considered in the accuracy measurement. Compared to MAPE, RMSE gives additional weight to larger absolute errors. Taken together, these three measures evaluate an assessment to be made of accuracy and precision of prediction reference. These measures are defined as follows:

Mean Percentage Error (MPE):

$$
M P E=\frac{1}{n} \sum_{i=1}^{n}\left(\frac{t t_{i}-t \hat{t}_{i}}{t t_{i}}\right) * 100 \%
$$

Mean Absolute Percentage Error (MAPE):

$$
\text { MAPE }=\frac{1}{n} \sum_{i=1}^{n}\left(\frac{\left|t t_{i}-t \hat{t}_{i}\right|}{t t_{i}}\right) * 100 \%
$$

Root Mean Square Error (RMSE):

$$
R M S E=\sqrt{\sum_{i=1}^{n}\left(\frac{\left|t t_{i}-t \hat{t}_{i}\right|^{2}}{n_{i}}\right)}
$$

Where,

$t t_{i}$ : observed travel time

$t \hat{t}_{i}:$ predicted travel time

$n:$ number of predicted time intervals.

\section{EXPERIMENTAL RESULTS AND DISCUSSION}

\section{Prediction results in Scenario 1}

\section{London case}

In this subsection, only travel time data collected from Link 1309 of the A40 road in central London LCAP system under normal traffic conditions is tested. The one-step ahead travel time prediction accuracy is compared using MPE, MAPE and RMSE metrics in Table 1.

The results in Table 1 indicate that, in general, the use of data smoothing improves prediction accuracy under normal traffic conditions in the London A40 corridor. For example, the value of MAPE by $\mathrm{kNN}$ without smoothing is $5.04 \%$, which is reduced to $3.76 \%$ using the two-stage framework with SSA, in other words, an improvement of $25.40 \%$. This difference is statistically significant at the $2.5 \%$ level (using the Wilcoxon signed-rank test). A similar result holds for the wavelet smoother and for the other prediction methods.

In general, the two smoothing approaches have similar effects. KNN, GM and RF predictors generally give rather similar results, which tend to be better than those from SVR. Overall, the best results are obtained using the SSA smoother linked to the RF predictor. Figure 5, 6 and 7 show the scatter-plot of predicted and observed travel time data, the error autocorrelation plot of predictions, the histogram of error distribution and the sample time-series plot between predicted and observed travel time of the RF method with the one-stage, twostage using SSA and two-stage using wavelets prediction frameworks. 


\section{Maidstone case}

In the dataset, the aggregated 5-minute travel time data was collected from Maidstone. Different traffic prediction frameworks with four different machine learning methods were evaluated. The results comparing one-step ahead prediction accuracy are given in Table 2.

The results in Table 2 indicate that, similar to the London A40 case, for the Maidstone A229 corridor the use of data smoothing improves prediction accuracy under normal traffic conditions. For example, the averaged value of MAPE without the proposed framework is $8.49 \%$, which is reduced to $5.65 \%$ with SSA smoothing and $5.68 \%$ with wavelets smoothing. Again as in London, the RF predictor emerges as marginally the best (although differences are in general small) and the differences between the SSA and wavelet smoothers are also small.

\section{Prediction results in Scenario 2}

\section{London case}

The results comparing the prediction accuracy of different frameworks with different machine learning methods during the abnormal period are shown in Table 3. The MPE, MAPE and RMSE values under abnormal traffic conditions were calculated based on the period starting 30 minutes before the occurrence of the abnormal event and finishing around 30 minutes after the clearance of the event. The assumption is that the traffic state might recover to normal conditions within 30 minutes. The abnormal event happened around 17:57 and cleared around 18:40.

As we expect, the overall level of error is larger in abnormal conditions than in normal conditions. But ever with these larger errors, a data smoothing structure using either SSA or wavelets improves the prediction accuracy. The absolute percentage errors were not analysed as the samples using Wilcoxon signed-rank test due to the insufficient number of samples under abnormal traffic conditions in this scenario. As with the earlier cases, the difference between the results obtained with SSA and wavelet smothers is small. Amongst the predictors, the kNN based method in general performed best.

Figure 8 shows the results for the kNN predictor using data during the incident period. The time-series plot shows the general underestimation of travel time during the beginning period of abnormal conditions and overestimation of travel time during the clearance period of abnormal conditions. The tendency during the abnormal periods is for a slight lag behind the actual travel times. When the traffic profile suddenly drops, the models cannot immediately detect this change. Compared to the SSA smoother, the wavelet method can more quickly detect the sudden change caused by incidents. However, it overestimates the peak value of the future travel time during incident conditions. More detailed information about abnormal events such as level of lane closure and severity of incidents and accidents may help models estimate the current traffic states and predict traffic variables more accurately during abnormal traffic conditions.

\section{Maidstone case}

Table 4 shows the prediction accuracy results for the Maidstone A229 corridor under abnormal conditions. As in the London A40 case, predictor accuracy is worse than under normal conditions but prediction is significant improved by the use of data smoothing. The average improvement in MAPE value is $27.76 \%$ for four different machine learning methods using the two-stage framework; while the improvement in the RMSE metric is $20.66 \%$.

Also as in the London A40 corridor example, the kNN based prediction method emerges as the best abnormal traffic conditions. Figure 9 is the time-series plot between predicted and observed travel time of the kNN method with and without prediction frameworks during the abnormal period. Similar to the wavelet performance of London A40 case, for Maidstone case, 
the wavelet method can more quickly detect the change of travel time when the incident started than the SSA smoother. However, it overestimates the peak values during incidents.

\section{CONCLUSIONS}

In this paper, we have explored the impact of alternative data smoothing/de-noising and alternative prediction methods on the quality of short-term travel time prediction, using the two-stage prediction framework introduced by Guo et al. (2013). The results indicate that under a range of different traffic conditions, the use of data smoothing/de-noising improves prediction accuracy regardless of the prediction method used and that this was true during normal traffic conditions and during incident conditions. The average improvement in prediction performance quantified using MAPE is $33.4 \%$ under normal traffic conditions and $21.7 \%$ during incidents. Similarly, the average improvement in RMSE is $22.6 \%$ and $18.0 \%$, during normal and incident conditions respectively. Moreover, it is also clear from the results that although there are some interesting differences in the performance of different prediction methods, the use of data smoothing in general has a much greater impact on prediction accuracy than the choice of a specific prediction method. Thus, it is concluded that the use of a data smoothing pre-processing step can be expected to improve the accuracy of prediction methods of traffic variables. Having said that, the impact of data smoothing on prediction accuracy for variables that typically exhibit a high level of variability, e.g. occupancy, needs to be further investigated; this is an area for future research.

Another interesting feature of the results is that there are appears to be little difference in the performance of the two data smoothing/de-nosing methods used - SSA and Wavelet decomposition. Amongst the prediction methods used, the Grey System method emerged as marginally the best under normal traffic conditions whereas under abnormal conditions, the kNN method was clearly superior. The latter can be attributed to the lazy learning approach employed by the kNN which enables it to quickly detect pattern changes and have the flexibility to match the best patterns from historical datasets.

We believe that the results of this work point strongly to the conclusion that the application of data smoothing methods should become a standard feature in short-term traffic prediction and that a fruitful direction for future research is likely to lie in the development of specialised smoothing and de-nosing techniques that are customised to the characteristics of specific traffic data sources and traffic state variables for prediction tasks.

\section{ACKNOWLEDGEMENT}

The authors would like to acknowledge the support from Transport for London (TfL) and Kent County Council (KCC) for providing the data used in this paper and Cisco for providing the financial support for this work.

\section{REFERENCES}

Ahmed, M., \& Cook, A. (1979). Analysis of freeway traffic time series data by using BoxJenkins techniques. Transportation Research Board, 722, 1-9.

Barclay, V., Bonner, R., \& Hamilton, I. (1997). Application of wavelet transforms to experimental spectra: smoothing, denoising, and data set compression. Analytical Chemistry, 69(1), 78-90.

Box, G. E. P., \& Jenkins, G. M. (1970). Time Series Analysis: Forecasting and Control. San Francisco: Holden-Day.

Breiman, L. (2001). Random forests. Machine learning, 45(1), 5-32. 
Brunet, D., Vrscay, E. R., \& Wang, Z. (2009). The Use Of Residuals In Image Denoising: Springer.

Chang, P. C., \& Fan, C. Y. (2008). A hybrid system integrating a wavelet and TSK fuzzy rules for stock price forecasting. Ieee Transactions on Systems Man and Cybernetics Part CApplications and Reviews, 38(6), 802-815.

Chapelle, O., \& Vapnik, V. (1999). Model selection for support vector machines. Advances in Neural Information Processing Systems, 12, 230-236.

Chen, H., \& Rakha, H. A. (2014). Real-time travel time prediction using particle filtering with a non-explicit state-transition model. Transportation Research Part C: Emerging Technologies, 43, Part 1(0), 112-126.

Cherkassky, V., \& Ma, Y. (2004). Practical selection of SVM parameters and noise estimation for SVM regression. Neural Networks, 17(1), 113-126.

Daubechies, I. (1990). The wavelet transform, time-frequency localization and signal analysis. IEEE Transactions on Information Theory, 36(5), 961-1005.

Davis, G. A., \& Nihan, N. L. (1991). Nonparametric regression and short-term freeway traffic forecasting. Journal of Transportation Engineering-ASCE, 117(2), 178-188.

Deng, J. L. (1989). Introduction to grey system theory. The Journal of Grey System, 1(1), 1-24.

Dougherty, M. (1995). A review of neural networks applied to transport. Transportation Research Part C: Emerging Technologies, 3(4), 247-260.

Ghil, M., \& Vautard, R. (1991). Interdecadal oscillations and the warming trend in global temperature time series. Nature, 350(6316), 324-327.

Golyandina, N., Nekrutkin, V., \& Zhigljavsky, A. (2001). Analysis Of Time Series Structure: SSA And Related Techniques (Vol. 90): Chapman \& Hall/CRC Press.

Guo, F., Krishnan, R., \& Polak, J. (2012). Short-term traffic prediction under normal and incident conditions using singular spectrum analysis and the k-nearest neighbour method. Proceedings of the 17th International Conference on Road Transport Information and Control (RTIC), London, UK, 1-6.

Guo, F., Krishnan, R., \& Polak, J. (2013). A computationally efficient two-stage method for short-term traffic prediction on urban roads. Transportation Planning and Technology, $36(1), 62-75$.

Hassani, H. (2007). Singular spectrum analysis: methodology and comparison. Journal of Data Science, 5(2), 239-257.

Hastie, T., Tibshirani, R., \& Friedman, J. H. (2001). The Elements of Statistical Learning: Data Mining, Inference, and Prediction. New York: Springer Verlag.

Kayacan, E., Ulutas, B., \& Kaynak, O. (2010). Grey system theory-based models in time series prediction. Expert Systems with Applications, 37(2), 1784-1789.

Kosarev, E., \& Pantos, E. (1983). Optimal smoothing of'noisy'data by fast Fourier transform. Journal of Physics E: Scientific Instruments, 16(6), 537-543.

Krishnan, R. (2008). Travel time estimation and forecasting on urban roads. (PhD Thesis), Centre for Transport Studies, Imperial College London.

Liaw, A., \& Wiener, M. (2002). Classification and regression by randomForest. $R$ news, 2(3), $18-22$.

Mineva, A., \& Popivanov, D. (1996). Method for single-trial readiness potential identification, based on singular spectrum analysis. Journal of Neuroscience Methods, 68(1), 91-99.

Okutani, I., \& Stephanedes, Y. (1984). Dynamic prediction of traffic volume through Kalman filtering theory. Transportation Research Part B: Methodological, 18(1), 1-11.

Robinson, S. (2005). The development and application of an urban link travel time model using data derived from inductive loop detectors. (PhD Thesis), Centre for Transport Studies, Imperial College London. 
Short, R., D, \& Fukunaga, K. (1981). The optimal distance measure for nearest neighbor classification. IEEE Transactions on Information Theory, 27(5), 622-627.

Sivapragasam, C., Liong, S. Y., \& Pasha, M. (2001). Rainfall and runoff forecasting with SSASVM approach. Journal of Hydroinformatics, 3(3), 141-152.

Smith, B. L., Williams, B. M., \& Keith Oswald, R. (2002). Comparison of parametric and nonparametric models for traffic flow forecasting. Transportation Research Part C: Emerging Technologies, 10(4), 303-321.

Stone, C. J. (1977). Consistent nonparametric regression. The Annals of Statistics, 5, 595-620.

Szeto, W. Y., Ghosh, B., Basu, B., \& O’Mahony, M. (2009). Multivariate traffic forecasting technique using Cell Transmission Model and SARIMA model. Journal of Transportation Engineering, 135(9), 658-667.

TfL. (2010). Travel in London Report 2. Retrieved 11 May 2012, from http://www.tfl.gov.uk/assets/downloads/Travel_in_London_Report_2.pdf

Torrence, C., \& Compo, G. P. (1998). A practical guide to wavelet analysis. Bulletin of the American Meteorological society, 79(1), 61-78.

Vapnik, V. (1995). The Nature of Statistical Learning Theory. New York: Springer Verlag.

Verikas, A., Gelzinis, A., \& Bacauskiene, M. (2011). Mining data with random forests: A survey and results of new tests. Pattern Recognition, 44(2), 330-349.

Vlahogianni, E. I., Golias, J. C., \& Karlaftis, M. G. (2004). Short-term traffic forecasting: Overview of objectives and methods. Transport Reviews, 24(5), 533-557.

Wu, C. H., Ho, J. M., \& Lee, D. T. (2004). Travel-time prediction with support vector regression. IEEE Transactions on Intelligent Transportation Systems, 5(4), 276-281.

Wu, Y., Chen, F., Lu, C., \& Yang, S. (2016). Urban traffic flow prediction using a spatiotemporal random effects model. Journal of Intelligent Transportation Systems, 20(3), 282-293.

Xiao, H., Sun, H., Ran, B., \& Oh, Y. (2003). Fuzzy-neural network traffic prediction framework with wavelet decomposition. Transportation Research Record, 1879, 1723.

Xie, Y., Zhang, Y., \& Ye, Z. (2007). Short-term traffic volume forecasting using Kalman filter with discrete wavelet decomposition. Computer Aided Civil and Infrastructure Engineering, 22(5), 326-334.

Yoon, B., \& Chang, H. (2014). Potentialities of data-driven nonparametric regression in urban signalized traffic flow forecasting. Journal of Transportation Engineering, 140(7), 04014027.

Zhang, Y., \& Zhang, Y. (2016). A comparative study of three multivariate short-term freeway traffic flow forecasting methods with missing data. Journal of Intelligent Transportation Systems, 20(3), 205-218. 
Table 1 Comparison of prediction accuracy of travel time on the A40 Road in London under normal traffic conditions

\begin{tabular}{|c|c|c|c|c|c|c|c|c|c|}
\hline \multirow{3}{*}{ Method } & \multicolumn{3}{|c|}{ MPE (\%) } & \multicolumn{3}{c|}{ MAPE (\%) } & \multicolumn{3}{c|}{ RMSE (sec) } \\
\cline { 2 - 11 } & $\begin{array}{c}\text { Without } \\
\text { Framew } \\
\text { ork }\end{array}$ & $\begin{array}{c}\text { Framew } \\
\text { ork } \\
\text { with } \\
\text { SSA }\end{array}$ & $\begin{array}{c}\text { Framew } \\
\text { ork } \\
\text { with } \\
\text { Wavelet } \\
\text { s }\end{array}$ & $\begin{array}{c}\text { Without } \\
\text { Framew } \\
\text { ork }\end{array}$ & $\begin{array}{c}\text { Framew } \\
\text { ork } \\
\text { with } \\
\text { SSA }\end{array}$ & $\begin{array}{c}\text { Framew } \\
\text { ork } \\
\text { with } \\
\text { Wavelet } \\
\text { s }\end{array}$ & $\begin{array}{c}\text { Without } \\
\text { Framew } \\
\text { ork }\end{array}$ & $\begin{array}{c}\text { Framew } \\
\text { ork } \\
\text { with } \\
\text { SSA }\end{array}$ & $\begin{array}{c}\text { Framew } \\
\text { ork } \\
\text { with } \\
\text { Wavelet } \\
\text { s }\end{array}$ \\
\hline kNN & 0.68 & -0.56 & -0.46 & 5.04 & 3.76 & 3.81 & 38.26 & 32.86 & 32.64 \\
\hline GM & 0.36 & 0.13 & 0.13 & 6.34 & 3.82 & 3.91 & 43.66 & 28.82 & 29.33 \\
\hline RF & 0.38 & 0.12 & 0.13 & 4.71 & 3.30 & 3.38 & 40.18 & 34.68 & 33.79 \\
\hline SVR & 1.55 & -0.86 & -0.61 & 6.07 & 3.95 & 3.51 & 51.66 & 46.09 & 44.37 \\
\hline Mean & 0.74 & -0.23 & -0.27 & 5.54 & 3.60 & 3.76 & 43.44 & 35.18 & 35.46 \\
\hline
\end{tabular}

Table 2 Comparison of prediction accuracy of travel time in Maidstone under normal traffic conditions

\begin{tabular}{|c|c|c|c|c|c|c|c|c|c|c|}
\hline & \multicolumn{3}{|c|}{ MPE (\%) } & \multicolumn{3}{c|}{ MAPE (\%) } & \multicolumn{3}{c|}{ RMSE (sec) } \\
\cline { 2 - 11 } Method & $\begin{array}{c}\text { Without } \\
\text { Framew } \\
\text { ork }\end{array}$ & $\begin{array}{c}\text { Framew } \\
\text { ork } \\
\text { with } \\
\text { SSA }\end{array}$ & $\begin{array}{c}\text { Framew } \\
\text { ork } \\
\text { with } \\
\text { Wavelet } \\
\text { S }\end{array}$ & $\begin{array}{c}\text { Without } \\
\text { Framew } \\
\text { ork }\end{array}$ & $\begin{array}{c}\text { Framew } \\
\text { ork } \\
\text { with } \\
\text { SSA }\end{array}$ & $\begin{array}{c}\text { Framew } \\
\text { ork } \\
\text { with } \\
\text { Wavelet } \\
\text { S }\end{array}$ & $\begin{array}{c}\text { Without } \\
\text { Framew } \\
\text { ork }\end{array}$ & $\begin{array}{c}\text { Framew } \\
\text { ork } \\
\text { with } \\
\text { SSA }\end{array}$ & $\begin{array}{c}\text { Framew } \\
\text { ork } \\
\text { with } \\
\text { Wavelet } \\
\text { S }\end{array}$ \\
\hline kNN & -1.39 & -0.85 & -0.47 & 9.28 & 5.12 & 5.31 & 103.14 & 69.54 & 55.66 \\
\hline GM & 0.99 & 0.84 & 0.55 & 9.54 & 6.15 & 6.32 & 103.26 & 79.57 & 78.94 \\
\hline RF & -1.52 & -0.45 & 0.31 & 7.00 & 5.34 & 5.37 & 81.65 & 64.88 & 69.54 \\
\hline SVR & -1.18 & -0.76 & -0.81 & 8.13 & 5.98 & 5.72 & 95.01 & 70.30 & 75.26 \\
\hline Mean & -0.78 & -0.30 & -0.11 & 8.49 & 5.65 & 5.68 & 95.77 & 71.08 & 69.85 \\
\hline
\end{tabular}

Table 3 Comparison of prediction accuracy on the A40 Road in London during abnormal period

\begin{tabular}{|c|c|c|c|c|c|c|c|c|c|}
\hline & \multicolumn{3}{|c|}{ MPE (\%) } & \multicolumn{3}{c|}{ MAPE (\%) } & \multicolumn{3}{c|}{ RMSE (sec) } \\
\cline { 2 - 10 } Method & $\begin{array}{c}\text { Without } \\
\text { Framew } \\
\text { ork }\end{array}$ & $\begin{array}{c}\text { Framew } \\
\text { ork } \\
\text { with } \\
\text { SSA }\end{array}$ & $\begin{array}{c}\text { Framew } \\
\text { ork } \\
\text { with } \\
\text { Wavelet } \\
\text { S }\end{array}$ & $\begin{array}{c}\text { Without } \\
\text { Framew } \\
\text { ork }\end{array}$ & $\begin{array}{c}\text { Framew } \\
\text { ork } \\
\text { with } \\
\text { SSA }\end{array}$ & $\begin{array}{c}\text { Framew } \\
\text { ork } \\
\text { with } \\
\text { Wavelet } \\
\text { s }\end{array}$ & $\begin{array}{c}\text { Without } \\
\text { Framew } \\
\text { ork }\end{array}$ & $\begin{array}{c}\text { Framew } \\
\text { ork } \\
\text { with } \\
\text { SSA }\end{array}$ & $\begin{array}{c}\text { Framew } \\
\text { ork } \\
\text { with } \\
\text { Wavelet } \\
\text { S }\end{array}$ \\
\hline kNN & -0.95 & -1.24 & -1.73 & 8.70 & 7.52 & 7.61 & 169.37 & 151.03 & 137.73 \\
\hline GM & 0.65 & 0.78 & 0.67 & 11.10 & 9.63 & 10.44 & 207.69 & 179.77 & 204.24 \\
\hline RF & 0.65 & -0.78 & -0.85 & 11.10 & 9.63 & 9.76 & 207.69 & 179.77 & 188.56 \\
\hline SVR & -1.23 & -0.93 & 0.81 & 11.06 & 8.04 & 8.16 & 211.50 & 156.13 & 150.88 \\
\hline Mean & -0.22 & -0.54 & -0.28 & 10.49 & 8.71 & 8.99 & 199.06 & 166.68 & 170.35 \\
\hline
\end{tabular}


Table 4 Comparison of prediction accuracy in Maidstone during abnormal period

\begin{tabular}{|c|c|c|c|c|c|c|c|c|c|}
\hline \multirow{2}{*}{$\begin{array}{c}\text { Method } \\
\text { s }\end{array}$} & \multicolumn{3}{|c|}{ MPE (\%) } & \multicolumn{3}{c|}{ MAPE (\%) } & \multicolumn{3}{c|}{ RMSE (sec) } \\
\cline { 2 - 11 } & $\begin{array}{c}\text { Wramew } \\
\text { ork }\end{array}$ & $\begin{array}{c}\text { Framew } \\
\text { ork } \\
\text { with } \\
\text { SSA }\end{array}$ & $\begin{array}{c}\text { Framew } \\
\text { ork } \\
\text { with } \\
\text { Wavelet } \\
\text { s }\end{array}$ & $\begin{array}{c}\text { Without } \\
\text { Framew } \\
\text { ork }\end{array}$ & $\begin{array}{c}\text { Framew } \\
\text { ork } \\
\text { with } \\
\text { SSA }\end{array}$ & $\begin{array}{c}\text { Framew } \\
\text { ork } \\
\text { with } \\
\text { Wavelet } \\
\text { s }\end{array}$ & $\begin{array}{c}\text { Without } \\
\text { Framew } \\
\text { ork }\end{array}$ & $\begin{array}{c}\text { Framew } \\
\text { ork } \\
\text { with } \\
\text { SSA }\end{array}$ & $\begin{array}{c}\text { Framew } \\
\text { ork } \\
\text { with } \\
\text { Wavelet } \\
\text { s }\end{array}$ \\
\hline kNN & -1.04 & -0.57 & -0.33 & 6.54 & 2.37 & 4.37 & 108.02 & 66.05 & 66.24 \\
\hline GM & -4.49 & -4.26 & -4.23 & 8.50 & 7.36 & 6.85 & 115.20 & 102.06 & 97.81 \\
\hline RF & -0.78 & 0.55 & 0.71 & 6.68 & 6.15 & 5.29 & 89.69 & 75.32 & 76.61 \\
\hline SVR & 0.23 & 0.33 & 0.32 & 6.55 & 3.72 & 4.69 & 93.73 & 78.32 & 82.86 \\
\hline Mean & -1.52 & -0.99 & -0.89 & 7.06 & 4.90 & 5.30 & 101.66 & 80.43 & 80.88 \\
\hline
\end{tabular}




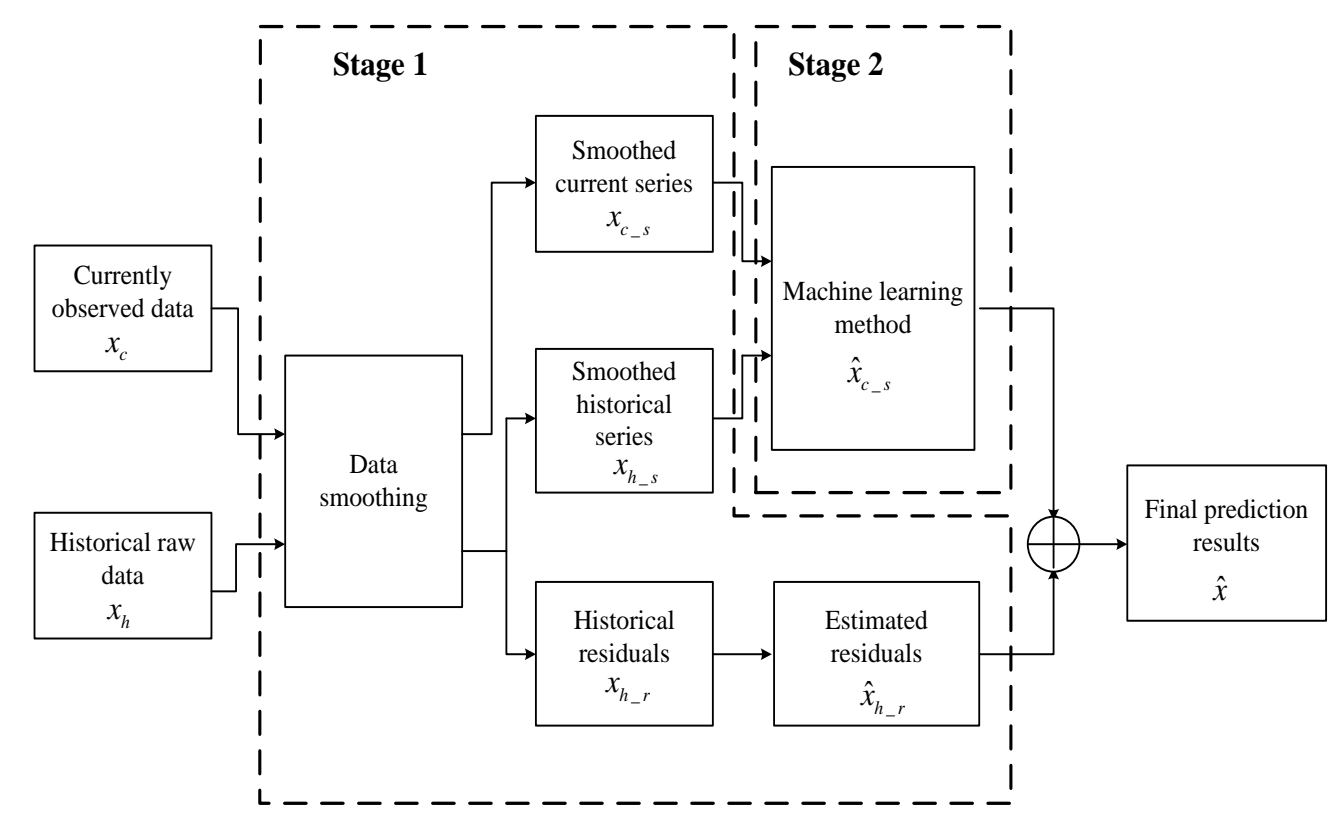

Figure 1 Flow-chart for the proposed two-stage prediction framework (Guo et al., 2013)
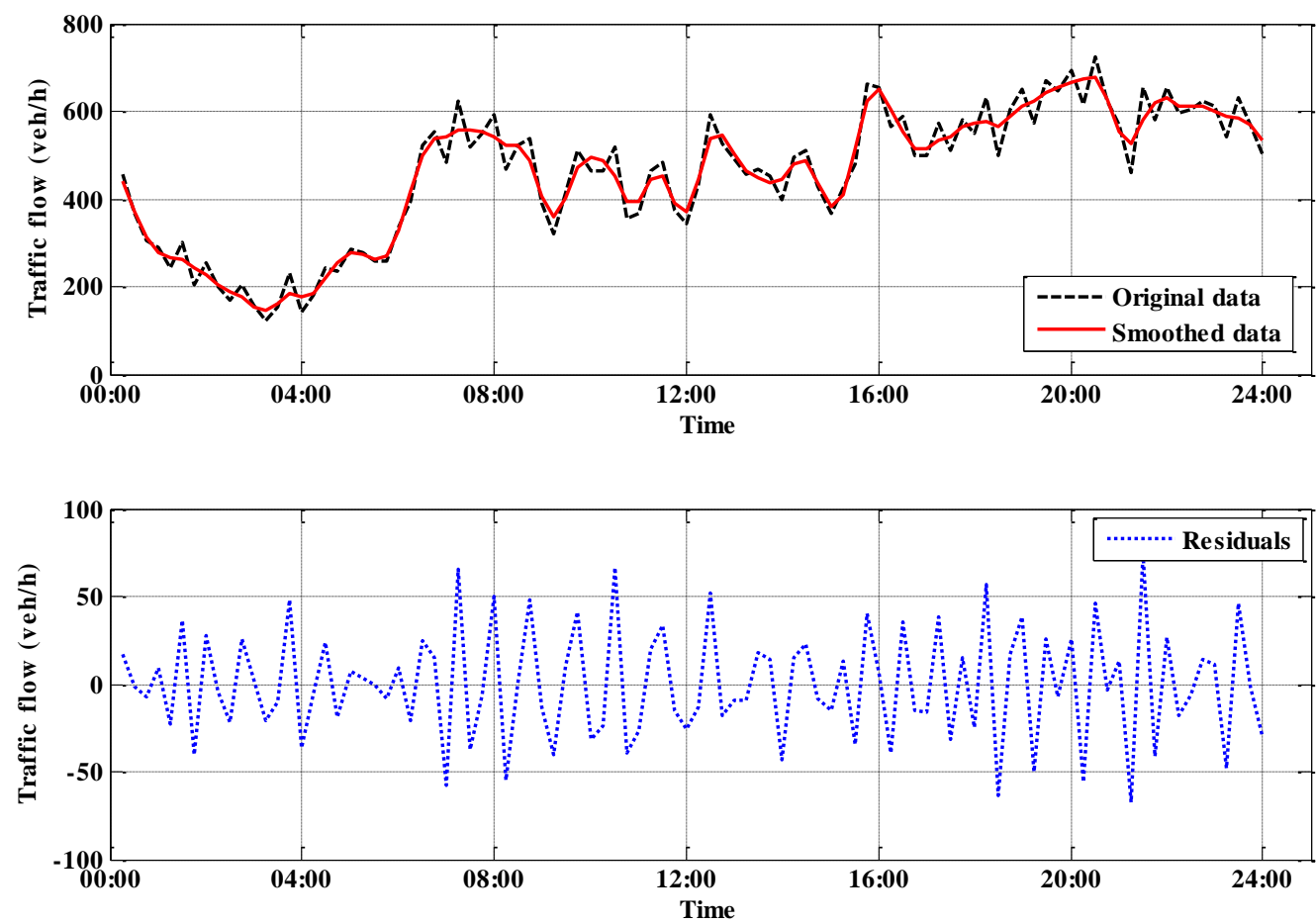

Figure 2 Traffic data, smoothed series and residuals 


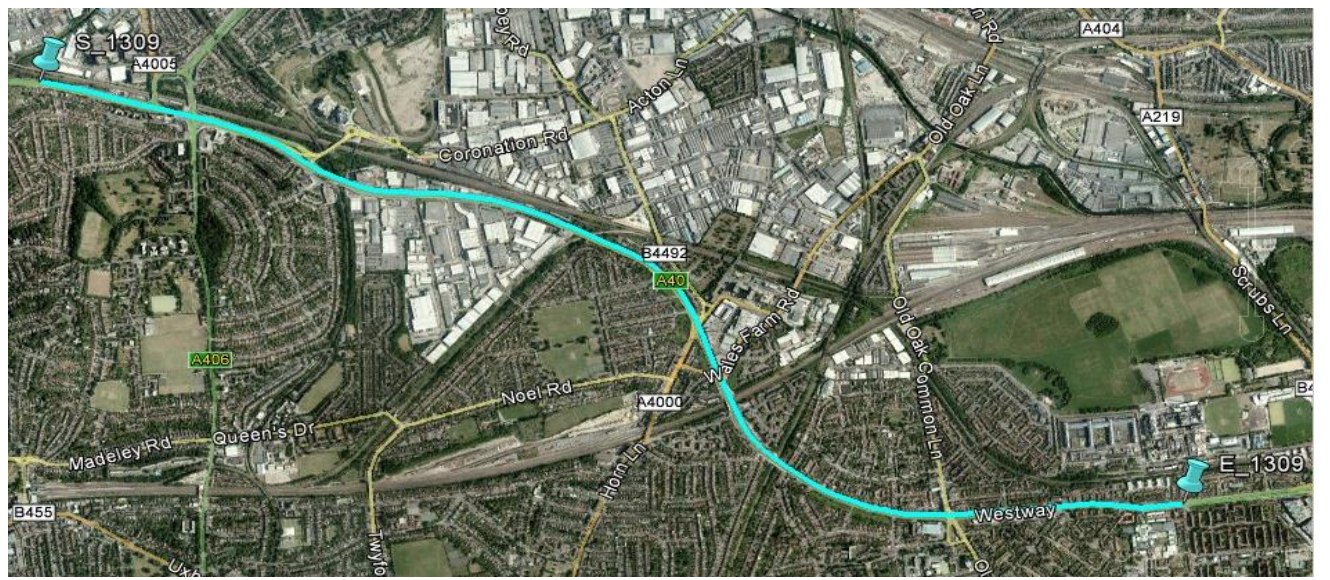

Figure 3 Link 1309 on the A40 road in London (Source: Google Earth)

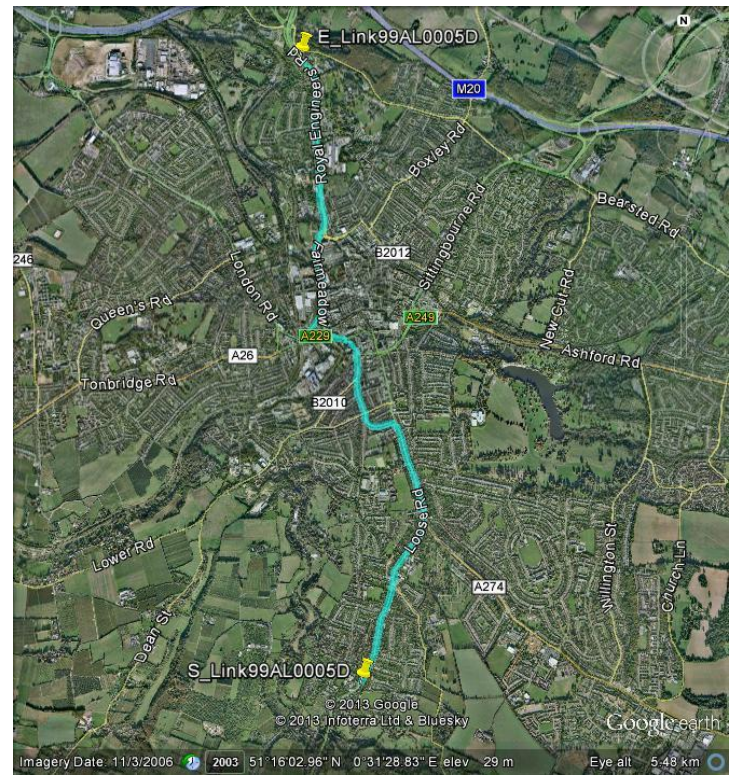

Figure 4 Selected Link 99AL0005D in Maidstone (Source: Google Earth) 

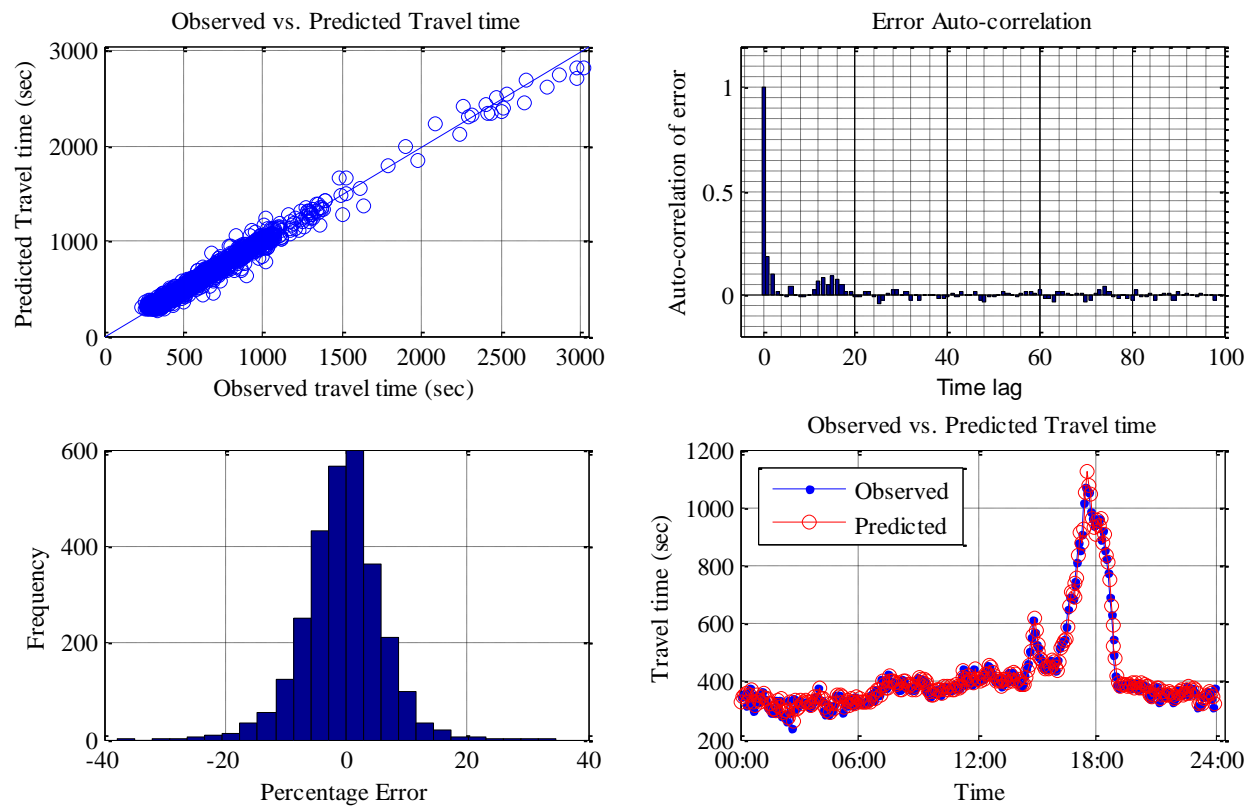

Figure 5 Travel time prediction performance using RF with the one-stage framework on the A40 road in London under normal traffic conditions
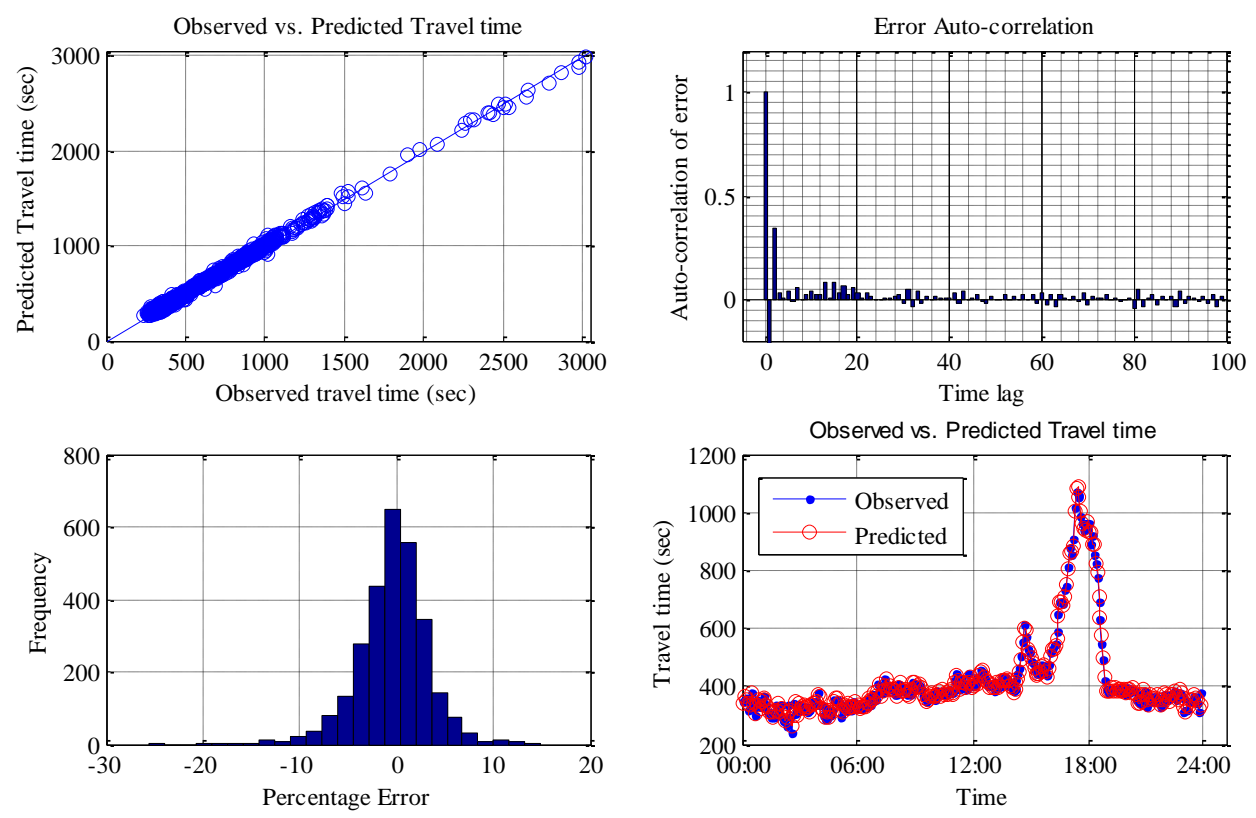

Figure 6 Travel time prediction performance using RF with the two-stage framework using SSA on the A40 road in London under normal traffic conditions 

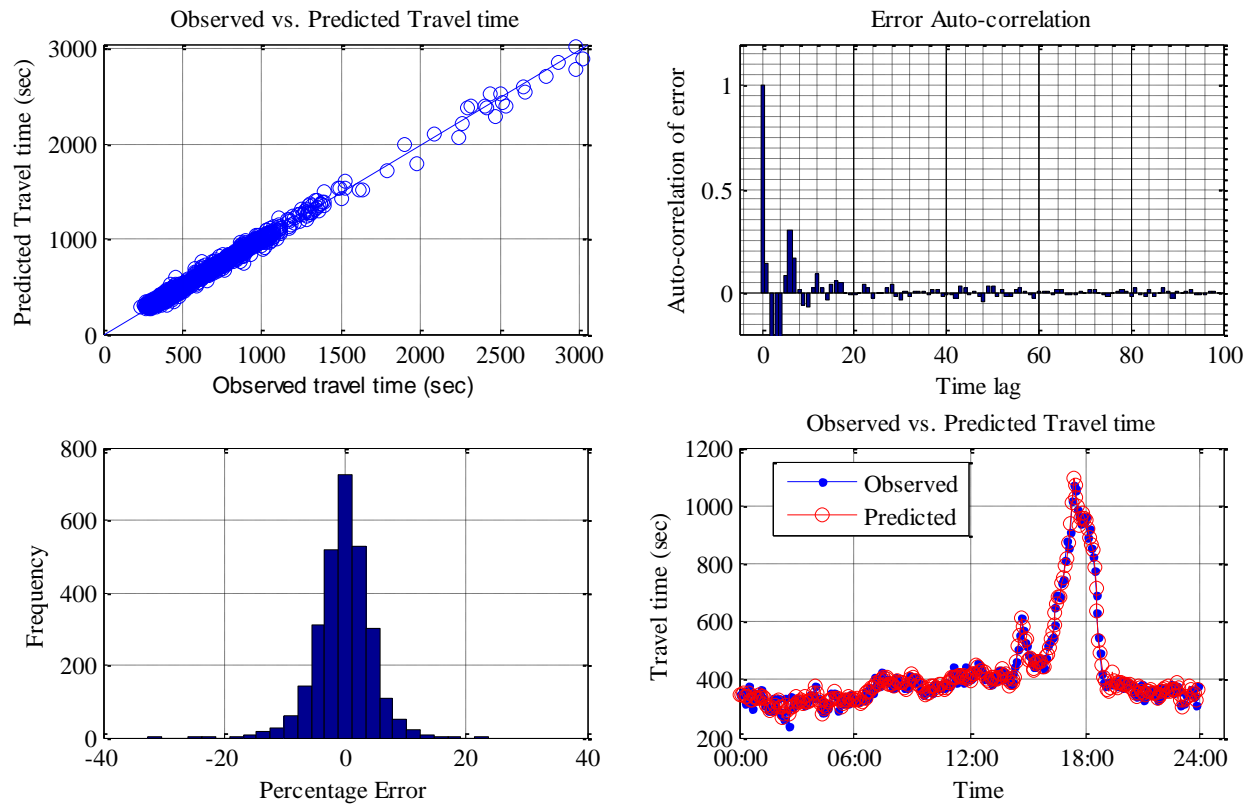

Figure 7 Travel time prediction performance using RF with the two-stage framework using Wavelets on the A40 road in London under normal traffic conditions

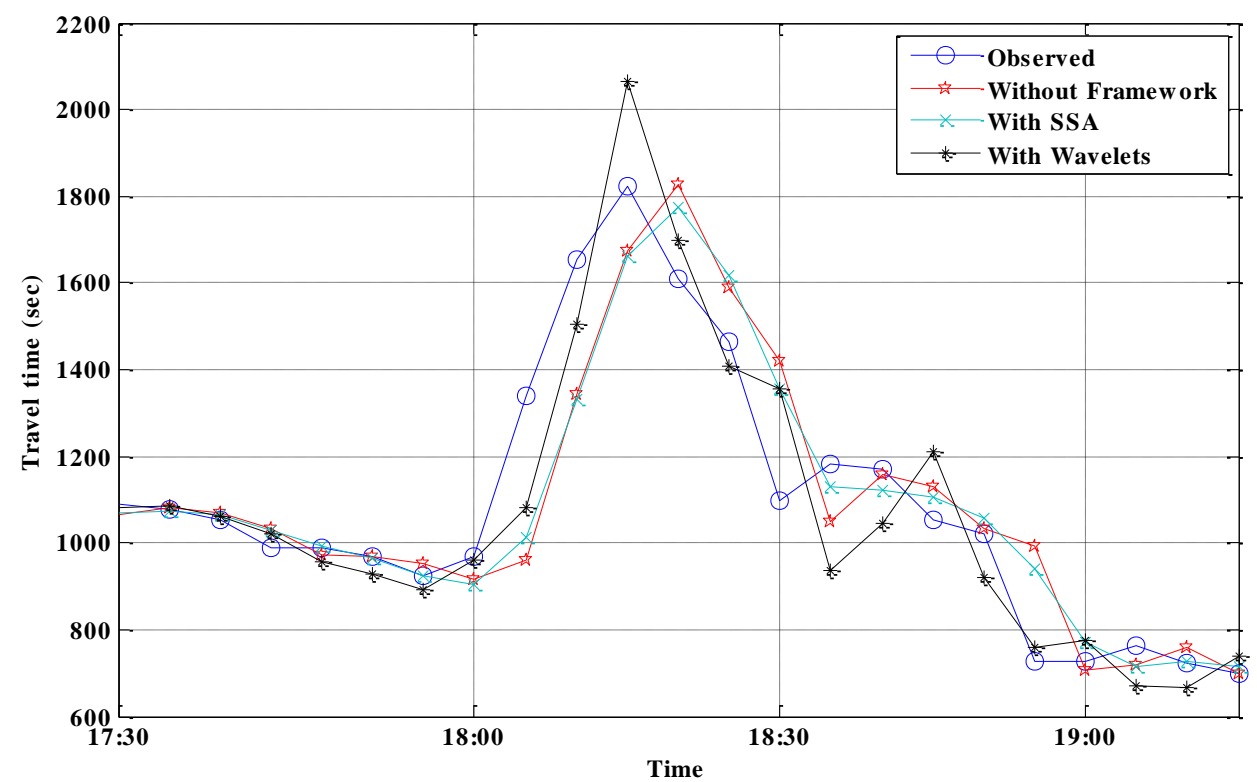

Figure 8 Comparison of observed and predicted travel time with and without prediction frameworks using the kNN based method on the A40 road in London during the abnormal period on the testing day 


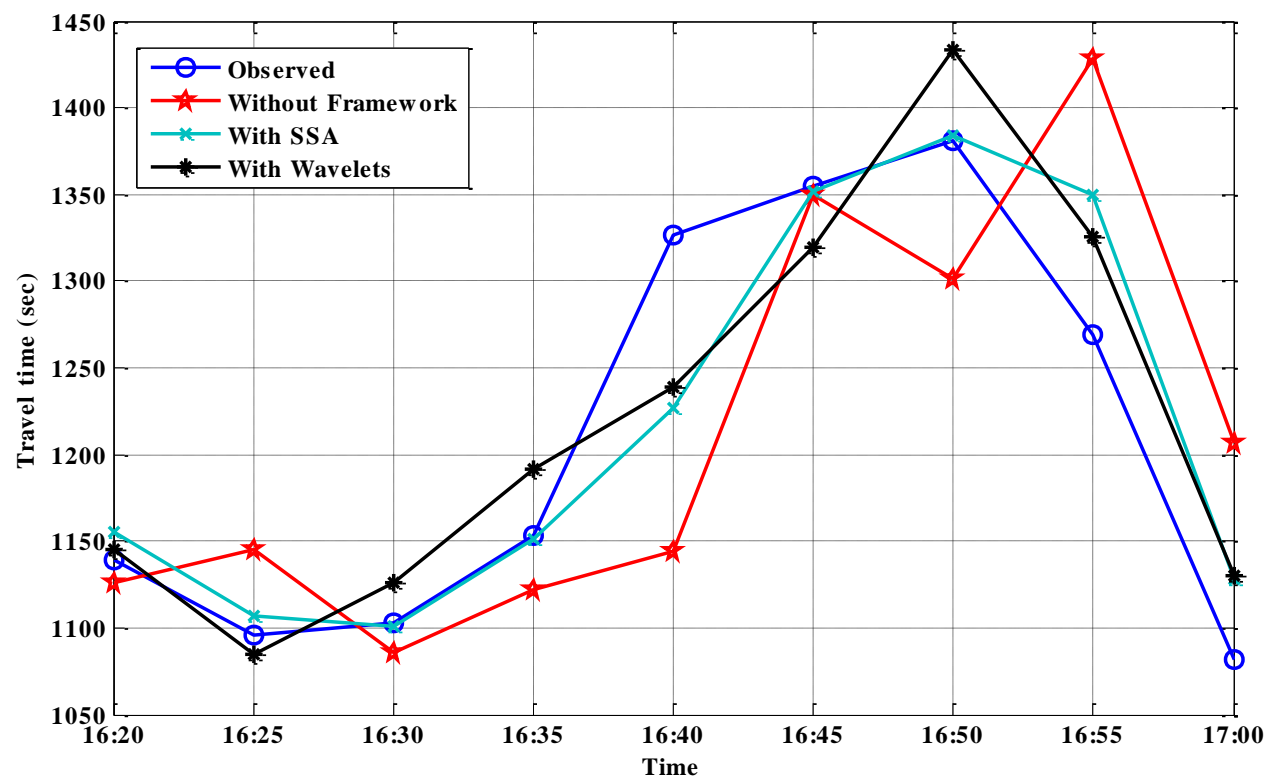

Figure 9 Comparison of observed and predicted travel time using three prediction frameworks with the kNN based method in Maidstone during the abnormal period 